Preprint, arXiv:0910.5667

\title{
ON SUMS OF BINOMIAL COEFFICIENTS MODULO $p^{2}$
}

\author{
ZHI-WEI SUN \\ Department of Mathematics, Nanjing University \\ Nanjing 210093, People's Republic of China \\ zwsun@nju.edu.cn \\ http://math.nju.edu.cn/ zwsun
}

ABstract. Let $p$ be an odd prime and $a$ be a positive integer. In this paper we investigate the sum $\sum_{k=0}^{p^{a}-1}\left(\begin{array}{c}h p^{a}-1 \\ k\end{array}\right)\left(\begin{array}{c}2 k \\ k\end{array}\right) / m^{k} \bmod p^{2}$, where $h, m$ are $p$-adic integers with $m \not \equiv 0(\bmod p)$. For example, we show that if $h \not \equiv 0(\bmod p)$ and $p^{a}>3$, then

$$
\begin{aligned}
& \sum_{k=0}^{p^{a}-1}\left(\begin{array}{c}
h p^{a}-1 \\
k
\end{array}\right)\left(\begin{array}{c}
2 k \\
k
\end{array}\right)\left(-\frac{h}{2}\right)^{k} \\
\equiv & \left(\frac{1-2 h}{p^{a}}\right)\left(1+h\left(\left(4-\frac{2}{h}\right)^{p-1}-1\right)\right)\left(\bmod p^{2}\right),
\end{aligned}
$$

where $(-)$ denotes the Jacobi symbol. Here is another remarkable congruence: If $p^{a}>3$ then

$$
\sum_{k=0}^{p^{a}-1}\left(\begin{array}{c}
p^{a}-1 \\
k
\end{array}\right)\left(\begin{array}{c}
2 k \\
k
\end{array}\right)(-1)^{k} \equiv 3^{p-1}\left(\frac{p^{a}}{3}\right)\left(\bmod p^{2}\right) .
$$

\section{INTRODUCTION}

Let $p$ be a prime. In 2006 H. Pan and Z. W. Sun [PS] proved that

$$
\sum_{k=0}^{p-1}\left(\begin{array}{c}
2 k \\
k+d
\end{array}\right) \equiv\left(\frac{p-d}{3}\right)(\bmod p) \quad \text { for } d=0, \ldots, p
$$

where $(-)$ is the Jacobi symbol. Later Sun and R. Tauraso [ST1, ST2] determined $\sum_{k=0}^{p^{a}-1}\left(\begin{array}{c}2 k \\ k\end{array}\right) / m^{k}$ and $\sum_{k=1}^{p-1}\left(\begin{array}{c}2 k \\ k\end{array}\right) /\left(k m^{k-1}\right)$ modulo $p$ via Lucas sequences, where $m$ is an integer not divisible by $p$ and $a$ is any positive

2010 Mathematics Subject Classification. Primary 11B65; Secondary 05A10, 11A07, 11B39, 11S99.

Keywords. Central binomial coefficients, congruences modulo prime powers.

Supported by the National Natural Science Foundation (grant 10871087) and the Overseas Cooperation Fund (grant 10928101) of China. 
integer. Recently Sun [S10a] was able to determine $\sum_{k=0}^{p^{a}-1}\left(\begin{array}{c}2 k \\ k\end{array}\right) / m^{k} \bmod$ $p^{2}$. See also [SSZ], [GZ] and [S10b] for related results on $p$-adic valuations.

In this paper we study a new kind of sums

$$
\sum_{k=0}^{p^{a}-1}\left(\begin{array}{c}
h p^{a}-1 \\
k
\end{array}\right) \frac{\left(\begin{array}{c}
2 k \\
k
\end{array}\right)}{m^{k}}
$$

modulo $p^{2}$, where $a \in \mathbb{Z}^{+}=\{1,2,3, \ldots\}$, and $h$ and $m$ are $p$-adic integers with $m \not \equiv 0(\bmod p)$.

For a prime $p$ we use $\mathbb{Z}_{p}$ to denote the ring of $p$-adic integers; if $h \in \mathbb{Z}_{p}$ and $h \not \equiv 0(\bmod p)$ then we denote the quotient $\left(h^{p-1}-1\right) / p \in \mathbb{Z}_{p}$ by $q_{p}(h)$ and call it a Fermat quotient. For $m, n \in \mathbb{N}=\{0,1,2, \ldots\}$, the Kronecker symbol $\delta_{m, n}$ takes 1 or 0 according as $m=n$ or not.

Now we state our main results and give some corollaries.

Theorem 1.1. Let $p$ be an odd prime and let $a \in \mathbb{Z}^{+}$. Let $h$ be a $p$-adic integer with $h \neq \equiv 0(\bmod p)$, and $\left(2 h \not \equiv 1(\bmod p)\right.$ or $\left.p^{a}>3\right)$. Then

$$
\begin{aligned}
& \sum_{k=0}^{p^{a}-1}\left(\begin{array}{c}
h p^{a}-1 \\
k
\end{array}\right)\left(\begin{array}{c}
2 k \\
k
\end{array}\right)\left(-\frac{h}{2}\right)^{k} \\
\equiv & \left(\frac{1-2 h}{p^{a}}\right)\left(1+h\left(\left(4-\frac{2}{h}\right)^{p-1}-1\right)\right)\left(\bmod p^{2}\right) .
\end{aligned}
$$

Corollary 1.1. Let $p$ be an odd prime and let $a \in \mathbb{Z}^{+}$. Then

$$
\sum_{k=0}^{p^{a}-1}\left(\begin{array}{c}
p^{a}-1 \\
k
\end{array}\right) \frac{\left(\begin{array}{c}
2 k \\
k
\end{array}\right)}{(-2)^{k}} \equiv(-1)^{\left(p^{a}-1\right) / 2} 2^{p-1}\left(\bmod p^{2}\right) .
$$

Proof. Simply apply Theorem 1.1 with $h=1$.

Remark 1.1. Let $m \in \mathbb{Z}$ and $n \in \mathbb{Z}^{+}$. Later we will show that

$$
\begin{aligned}
& \sum_{k=0}^{n-1}\left(\begin{array}{c}
n-1 \\
k
\end{array}\right)\left(\begin{array}{c}
2 k \\
k
\end{array}\right)(-1)^{k} m^{n-1-k} \\
= & \sum_{k=0}^{\lfloor(n-1) / 2\rfloor}\left(\begin{array}{c}
n-1 \\
k
\end{array}\right)\left(\begin{array}{c}
n-1-k \\
k
\end{array}\right)(m-2)^{n-1-2 k} .
\end{aligned}
$$

Thus, for any prime $p>3$, by applying Morley's congruence (cf. $[\mathrm{M}],[\mathrm{C}]$ and $[\mathrm{P}])$

we get

$$
\left(\begin{array}{c}
p-1 \\
(p-1) / 2
\end{array}\right) \equiv(-1)^{(p-1) / 2} 4^{p-1}\left(\bmod p^{3}\right)
$$

$$
\sum_{k=0}^{p-1}\left(\begin{array}{c}
p-1 \\
k
\end{array}\right) \frac{\left(\begin{array}{c}
2 k \\
k
\end{array}\right)}{(-2)^{k}} \equiv(-1)^{(p-1) / 2} 2^{p-1}\left(\bmod p^{3}\right)
$$

which is a refinement of (1.2) in the case $a=1$. 
Corollary 1.2. Let $p>3$ be a prime and let $a \in \mathbb{Z}^{+}$. Then

$$
\sum_{k=0}^{p^{a}-1}\left(\begin{array}{c}
2 p^{a}-1 \\
k
\end{array}\right)\left(\begin{array}{c}
2 k \\
k
\end{array}\right)(-1)^{k} \equiv\left(\frac{p^{a}}{3}\right)\left(2 \times 3^{p-1}-1\right)\left(\bmod p^{2}\right)
$$

and

$$
\sum_{k=0}^{p^{a}-1}\left(\begin{array}{c}
p^{a}+k \\
k
\end{array}\right) \frac{\left(\begin{array}{c}
2 k \\
k
\end{array}\right)}{2^{k}} \equiv\left(\frac{3}{p^{a}}\right)\left(1-p\left(q_{p}(2)+q_{p}(3)\right)\left(\bmod p^{2}\right) .\right.
$$

Proof. Just put $h=2$ and $h=-1$ in (1.1) and note that $\left(\begin{array}{c}-x \\ k\end{array}\right)=$ $(-1)^{k}\left(\begin{array}{c}x+k-1 \\ k\end{array}\right)$.

Corollary 1.3. Let $p$ be an odd prime and let $a \in \mathbb{Z}^{+}$. Then

$$
\sum_{k=0}^{p^{a}-1}\left(\begin{array}{c}
2 p^{a}+k \\
k
\end{array}\right)\left(\begin{array}{c}
2 k \\
k
\end{array}\right)(-1)^{k} \equiv\left(\frac{p^{a}}{5}\right)\left(3-2 \times 5^{p-1}\right)\left(\bmod p^{2}\right) .
$$

Proof. Simply apply (1.1) with $h=-2$.

Now we need to introduce Lucas sequences.

Let $A, B \in \mathbb{Z}$. The Lucas sequences $u_{n}=u_{n}(A, B)(n \in \mathbb{N})$ and $v_{n}=v_{n}(A, B)(n \in \mathbb{N})$ are defined by

$$
u_{0}=0, u_{1}=1 \text {, and } u_{n+1}=A u_{n}-B u_{n-1}(n=1,2,3, \ldots)
$$

and

$$
v_{0}=2, v_{1}=A \text {, and } v_{n+1}=A v_{n}-B v_{n-1}(n=1,2,3, \ldots) .
$$

The characteristic equation $x^{2}-A x+B=0$ has two roots

$$
\alpha=\frac{A+\sqrt{\Delta}}{2} \text { and } \beta=\frac{A+\sqrt{\Delta}}{2},
$$

where $\Delta=A^{2}-4 B$. It is well known that for any $n \in \mathbb{N}$ we have

$$
u_{n}=\sum_{0 \leqslant k<n} \alpha^{k} \beta^{n-1-k} \text { and } v_{n}=\alpha^{n}+\beta^{n} .
$$

If $p$ is a prime then

$$
v_{p}=\alpha^{p}+\beta^{p} \equiv(\alpha+\beta)^{p}=A^{p} \equiv A(\bmod p) .
$$

It is also known that

$$
u_{p} \equiv\left(\frac{\Delta}{p}\right)(\bmod p) \text { and } u_{p-\left(\frac{\Delta}{p}\right)} \equiv 0(\bmod p)
$$

for any prime $p$ not dividing 2B. (See, e.g., [S10a, Lemma 2.3].) The reader may consult [S06] for connections between Lucas sequences and quadratic fields.

Our following result is more general than Theorem 1.1. 
Theorem 1.2. Let $p$ be an odd prime and $m \in \mathbb{Z}$ with $p \nmid m$. Set $\Delta=$ $m(m-4)$ and let $h \in \mathbb{Z}_{p}$. Then we have

$$
\begin{aligned}
& \sum_{k=0}^{p^{a}-1}\left(\begin{array}{c}
h p^{a}-1 \\
k
\end{array}\right) \frac{\left(\begin{array}{c}
2 k \\
k
\end{array}\right)}{(-m)^{k}} \\
\equiv & \left(\frac{\Delta}{p^{a-1}}\right)\left(1-\frac{h m}{2}\right) u_{p-\left(\frac{\Delta}{p}\right)}(m-2,1) \\
& +\left(\frac{\Delta}{p^{a}}\right)\left(1+h\left((m-4)^{p-1}-1\right)\right) \\
& - \begin{cases}h(m-4)\left(\bmod p^{2}\right) & \text { if } p^{a}=3 \text { and } 3 \mid m-1, \\
0\left(\bmod p^{2}\right) & \text { otherwise. }\end{cases}
\end{aligned}
$$

In particular, if $h m \equiv 2(\bmod p)$ then

$$
\begin{aligned}
& \sum_{k=0}^{p^{a}-1}\left(\begin{array}{c}
h p^{a}-1 \\
k
\end{array}\right) \frac{\left(\begin{array}{c}
2 k \\
k
\end{array}\right)}{(-m)^{k}} \\
\equiv & \left(\frac{\Delta}{p^{a}}\right)\left(1+h\left((m-4)^{p-1}-1\right)\right) \\
& + \begin{cases}m-4\left(\bmod p^{2}\right) & \text { if } p^{a}=3 \text { and } 3 \mid m-1, \\
0\left(\bmod p^{2}\right) & \text { otherwise. }\end{cases}
\end{aligned}
$$

Corollary 1.4. Let $p$ be an odd prime and let $a \in \mathbb{Z}^{+}$with $p^{a}>3$. Then

$$
\sum_{k=0}^{p^{a}-1}\left(\begin{array}{c}
p^{a}-1 \\
k
\end{array}\right)\left(\begin{array}{c}
2 k \\
k
\end{array}\right)(-1)^{k} \equiv 3^{p-1}\left(\frac{p^{a}}{3}\right)\left(\bmod p^{2}\right)
$$

and

$$
\sum_{k=0}^{p^{a}-1}\left(\begin{array}{c}
p^{a}-1 \\
k
\end{array}\right) \frac{\left(\begin{array}{c}
2 k \\
k
\end{array}\right)}{(-3)^{k}} \equiv\left(\frac{p^{a}}{3}\right)\left(\bmod p^{2}\right) .
$$

Proof. Just apply (1.7) with $h=1$ and $m \in\{1,3\}$ and note that $(-1)^{n-1} u_{n}(1,1)=u_{n}(-1,1)=\left(\frac{n}{3}\right)$ for $n \in \mathbb{N}$.

Corollary 1.5. Let $p \neq 2,5$ be a prime and let $a \in \mathbb{Z}^{+}$. Then

$$
\sum_{k=0}^{p^{a}-1}\left(\begin{array}{c}
p^{a}-1 \\
k
\end{array}\right)\left(\begin{array}{c}
2 k \\
k
\end{array}\right) \equiv\left(\frac{p^{a}}{5}\right)\left(5^{p-1}-3 F_{p-\left(\frac{p}{5}\right)}\right)\left(\bmod p^{2}\right)
$$

and

$$
\sum_{k=0}^{p^{a}-1}\left(\begin{array}{c}
p^{a}-1 \\
k
\end{array}\right) \frac{\left(\begin{array}{c}
2 k \\
k
\end{array}\right)}{(-5)^{k}} \equiv\left(\frac{p^{a}}{5}\right)\left(1-3 F_{p-\left(\frac{p}{5}\right)}\right)\left(\bmod p^{2}\right),
$$


where $\left\{F_{n}\right\}_{n \geqslant 0}$ is the well-known Fibonacci sequence defined by

$$
F_{0}=0, F_{1}=1 \text {, and } F_{n+1}=F_{n}+F_{n-1}(n=1,2,3, \ldots) \text {. }
$$

Proof. Observe that

$$
(-1)^{n-1} u_{n}(-3,1)=u_{n}(3,1)=F_{2 n}=F_{n} L_{n},
$$

where $L_{n}=v_{n}(1,-1)$. By [SS, Corollary 1] (or the proof of Corollary 1.3 of $[\mathrm{ST} 1])$, if $p \neq 2,5$ then $L_{p-\left(\frac{p}{5}\right)} \equiv 2\left(\frac{p}{5}\right)\left(\bmod p^{2}\right)$. In view of this, if we apply (1.7) with $h=1$ and $m \in\{-1,5\}$ the we obtain the desired result.

To conclude this section we raise four conjectures.

Conjecture 1.1. Let $p$ be an odd prime and let $h$ be an integer with $2 h-1 \equiv 0(\bmod p)$. If $a \in \mathbb{Z}^{+}$and $p^{a}>3$, then

$$
\sum_{k=0}^{p^{a}-1}\left(\begin{array}{c}
h p^{a}-1 \\
k
\end{array}\right)\left(\begin{array}{c}
2 k \\
k
\end{array}\right)\left(-\frac{h}{2}\right)^{k} \equiv 0\left(\bmod p^{a+1}\right) .
$$

Also, for any $n \in \mathbb{Z}^{+}$we have

$$
\frac{1}{n} \sum_{k=0}^{n-1}\left(\begin{array}{c}
h n-1 \\
k
\end{array}\right)\left(\begin{array}{c}
2 k \\
k
\end{array}\right)\left(-\frac{h}{2}\right)^{k} \in \mathbb{Z}_{p}
$$

Conjecture 1.2. Let $p>3$ be a prime. Then

$$
\sum_{k=0}^{p-1}\left(\begin{array}{c}
p-1 \\
k
\end{array}\right)\left(\begin{array}{c}
2 k \\
k
\end{array}\right)\left((-1)^{k}-(-3)^{-k}\right) \equiv\left(\frac{p}{3}\right)\left(3^{p-1}-1\right)\left(\bmod p^{3}\right) .
$$

Conjecture 1.3. Let $p$ be a prime with $p \equiv \pm 1(\bmod 12)$. Then

$$
\sum_{k=0}^{p-1}\left(\begin{array}{c}
p-1 \\
k
\end{array}\right)\left(\begin{array}{c}
2 k \\
k
\end{array}\right)(-1)^{k} u_{k}(4,1) \equiv(-1)^{(p-1) / 2} u_{p-1}(4,1)\left(\bmod p^{3}\right) .
$$

Conjecture 1.4. Let $p$ be a prime with $p \equiv \pm 1(\bmod 8)$. Then

$$
\sum_{k=0}^{p-1}\left(\begin{array}{c}
p-1 \\
k
\end{array}\right)\left(\begin{array}{c}
2 k \\
k
\end{array}\right) \frac{u_{k}(4,2)}{(-2)^{k}} \equiv(-1)^{(p-1) / 2} u_{p-1}(4,2)\left(\bmod p^{3}\right) .
$$

We remark that the author could prove the congruences in Conjectures 1.2-1.4 modulo $p^{2}$.

In the next section we provide some lemmas. Section 3 is devoted to our proofs of Theorems 1.1-1.2 and (1.3). 


\section{Some Lemmas}

For $n \in \mathbb{N}$ we set $H_{n}=\sum_{0<k \leqslant n} 1 / k$.

Lemma 2.1. Let $p$ be an odd prime and let $m \in \mathbb{Z}$ with $p \nmid m$. If $p \mid m-4$ then

$$
\sum_{k=1}^{p^{a}-1} \frac{p^{a-1} H_{k}}{m^{k}}\left(\begin{array}{c}
2 k \\
k
\end{array}\right) \equiv 2 \delta_{a, 1}(\bmod p) .
$$

When $m \not \equiv 4(\bmod p)$, we have

$$
\sum_{k=1}^{p^{a}-1} \frac{p^{a-1} H_{k}}{m^{k}}\left(\begin{array}{c}
2 k \\
k
\end{array}\right) \equiv-\left(\frac{m(m-4)}{p^{a}}\right) \sum_{k=1}^{p-1} \frac{\left(\begin{array}{c}
2 k \\
k
\end{array}\right)}{k(4-m)^{k}}(\bmod p) .
$$

Proof. For $k=1, \ldots,\left(p^{a}-1\right) / 2$, we have

$$
\begin{aligned}
\frac{\left(\begin{array}{c}
\left(\begin{array}{c}
a \\
k
\end{array}\right) / 2 \\
k
\end{array}\right)}{\left(\begin{array}{c}
2 k \\
k
\end{array}\right) /(-4)^{k}} & =\frac{\left(\begin{array}{c}
\left(p^{a}-1\right) / 2 \\
k
\end{array}\right)}{\left(\begin{array}{c}
-1 / 2 \\
k
\end{array}\right)}=\prod_{j=1}^{k} \frac{\left(p^{a}-1\right) / 2-j+1}{-1 / 2-j+1} \\
& =\prod_{j=1}^{k}\left(1-\frac{p^{a}}{2 j-1}\right) \equiv 1(\bmod p) .
\end{aligned}
$$

If $k \in\left\{\left(p^{a}+1\right) / 2, \ldots, p^{a}-1\right\}$, then $2 k-p^{a} \in\{1, \ldots, k-1\}$ and hence

$$
\left(\begin{array}{c}
2 k \\
k
\end{array}\right)=\left(\begin{array}{c}
p^{a}+\left(2 k-p^{a}\right. \\
k
\end{array}\right) \equiv\left(\begin{array}{c}
p^{a} \\
0
\end{array}\right)\left(\begin{array}{c}
2 k-p^{a} \\
k
\end{array}\right)=0(\bmod p)
$$

with the help of Lucas' congruence. So, for any $k=0, \ldots, p^{a}-1$ we have

$$
\left(\begin{array}{c}
2 k \\
k
\end{array}\right) \equiv(-4)^{k}\left(\begin{array}{c}
\left(p^{a}-1\right) / 2 \\
k
\end{array}\right)(\bmod p) .
$$

Note also that

$$
p^{a-1} H_{k}=\sum_{j=1}^{k} \frac{p^{a-1}}{j} \in \mathbb{Z}_{p} \quad \text { for every } k=1, \ldots, p^{a}-1 .
$$

Therefore

$$
\sum_{k=1}^{p^{a}-1} \frac{p^{a-1} H_{k}}{m^{k}}\left(\begin{array}{c}
2 k \\
k
\end{array}\right) \equiv \sum_{k=1}^{\left(p^{a}-1\right) / 2}\left(\begin{array}{c}
\left(p^{a}-1\right) / 2 \\
k
\end{array}\right)\left(-\frac{4}{m}\right)^{k}\left(p^{a-1} H_{k}\right)(\bmod p) .
$$


For each $k \in \mathbb{N}$ clearly

$$
\begin{aligned}
H_{k} & =\sum_{0<j \leqslant k} \int_{0}^{1} x^{j-1} d x=\int_{0}^{1} \sum_{0<j \leqslant k} x^{j-1} d x \\
& =\int_{0}^{1} \frac{1-x^{k}}{1-x} d x=\int_{0}^{1} \frac{1-(1-t)^{k}}{t} d t .
\end{aligned}
$$

Thus

$$
\sum_{k=1}^{p^{a}-1} \frac{p^{a-1} H_{k}}{m^{k}}\left(\begin{array}{c}
2 k \\
k
\end{array}\right) \equiv p^{a-1} \Sigma(\bmod p),
$$

where

$$
\begin{aligned}
\Sigma & :=\int_{0}^{1} \sum_{k=0}^{\left(p^{a}-1\right) / 2}\left(\begin{array}{c}
\left(p^{a}-1\right) / 2 \\
k
\end{array}\right)\left(-\frac{4}{m}\right)^{k} \frac{1-(1-t)^{k}}{t} d t \\
& =\int_{0}^{1} \frac{(1-4 / m)^{\left(p^{a}-1\right) / 2}-(1-(1-t) 4 / m)^{\left(p^{a}-1\right) / 2}}{t} d t \\
& =-\sum_{k=1}^{\left(p^{a}-1\right) / 2}\left(\begin{array}{c}
\left(p^{a}-1\right) / 2 \\
k
\end{array}\right)\left(1-\frac{4}{m}\right)^{\left(p^{a}-1\right) / 2-k} \int_{0}^{1}\left(\frac{4 t}{m}\right)^{k} \frac{d t}{t} \\
& =-\frac{1}{m^{\left(p^{a}-1\right) / 2}} \sum_{k=1}^{\left(p^{a}-1\right) / 2}\left(\begin{array}{c}
\left(p^{a}-1\right) / 2 \\
k
\end{array}\right) \frac{4^{k}}{k}(m-4)^{\left(p^{a}-1\right) / 2-k} .
\end{aligned}
$$

If $m \equiv 4(\bmod p)$, then

$$
p^{a-1} \Sigma \equiv-\frac{1}{m^{\left(p^{a}-1\right) / 2}} \cdot \frac{p^{a-1}}{\left(p^{a}-1\right) / 2} 4^{\left(p^{a}-1\right) / 2} \equiv 2 \delta_{a, 1}(\bmod p)
$$

and hence (2.1) holds.

Now assume that $m \not \equiv 4(\bmod p)$. In view of $(2.3)$,

$$
\begin{aligned}
p^{a-1} \Sigma & \equiv-\frac{(m(m-4))^{\left(p^{a}-1\right) / 2}}{m^{p^{a}-1}} \sum_{k=1}^{p^{a}-1}\left(\begin{array}{c}
2 k \\
k
\end{array}\right) \frac{(-1)^{k} p^{a-1}}{k(m-4)^{k}} \\
& \equiv-\left(\frac{m(m-4)}{p^{a}}\right) p^{a-1} \sum_{k=1}^{p^{a}-1} \frac{\left(\begin{array}{c}
2 k \\
k
\end{array}\right)}{k(4-m)^{k}}(\bmod p)
\end{aligned}
$$

So it suffices to prove that

$$
p^{a-1} \sum_{k=1}^{p^{a}-1} \frac{\left(\begin{array}{c}
2 k \\
k
\end{array}\right)}{k n^{k}} \equiv \sum_{k=1}^{p-1} \frac{\left(\begin{array}{c}
2 k \\
k
\end{array}\right)}{k n^{k}}(\bmod p)
$$


for any $n \in \mathbb{Z}$ with $p \nmid n$. If $p^{a-1} \nmid k$ then $p^{a-1} / k \equiv 0(\bmod p)$. Therefore

$$
p^{a-1} \sum_{k=1}^{p^{a}-1} \frac{\left(\begin{array}{c}
2 k \\
k
\end{array}\right)}{k n^{k}} \equiv p^{a-1} \sum_{j=1}^{p-1} \frac{\left(\begin{array}{c}
2 p^{a-1} j \\
p^{a-1} j
\end{array}\right)}{p^{a-1} j n^{p^{a-1} j}} \equiv \sum_{j=1}^{p-1} \frac{\left(\begin{array}{c}
2 j \\
j
\end{array}\right)}{j n^{j}}(\bmod p)
$$

in view of the Lucas congruence (cf. [St, p. 44]).

So far we have completed the proof of Lemma 2.1.

Lemma 2.2 (Sun $[\mathrm{S} 10 \mathrm{a}])$. Let $p$ be an odd prime and let $a \in \mathbb{Z}^{+}$. Let $m$ be any integer not divisible by $p$ and set $\Delta=m(m-4)$. Then we have

$$
\sum_{k=0}^{p^{a}-1} \frac{\left(\begin{array}{c}
2 k \\
k
\end{array}\right)}{m^{k}} \equiv\left(\frac{\Delta}{p^{a}}\right)+\left(\frac{\Delta}{p^{a-1}}\right) u_{p-\left(\frac{\Delta}{p}\right)}(m-2,1)\left(\bmod p^{2}\right)
$$

Lemma 2.3 (Sun and Tauraso [ST1]). Let $p$ be a prime and let $m$ be an integer not divisible by $p$. Then we have

$$
\frac{1}{2} \sum_{k=1}^{p-1}(-1)^{k} \frac{\left(\begin{array}{c}
2 k \\
k
\end{array}\right)}{k m^{k-1}} \equiv \frac{m^{p}-v_{p}(m,-m)}{p}(\bmod p) .
$$

Lemma 2.4. Let $p$ be an odd prime and let $m \in \mathbb{Z}$ with $\Delta=m(m-4) \not \equiv$ $0(\bmod p)$. Then

$$
\begin{aligned}
& \frac{2}{m-4} \cdot \frac{v_{p}(m-4,4-m)-(m-4)^{p}}{p} \\
\equiv & \frac{m}{2}\left(\frac{\Delta}{p}\right) \frac{u_{p-\left(\frac{\Delta}{p}\right)}(m-2,1)}{p}-q_{p}(m-4)(\bmod p) .
\end{aligned}
$$

Proof. (i) Let us first show the equality

$$
\frac{v_{2 n+1}(m-4,4-m)}{(m-4)^{n+1}}=\frac{u_{2 n+1}(m, m)}{m^{n}}
$$

for $n=0,1,2, \ldots$ Clearly both sides of (2.3) coincide with 1 when $n=0$. Note that

$$
\begin{aligned}
& \frac{v_{3}(m-4,4-m)}{(m-4)^{2}}=\frac{v_{2}(m-4,4-m)+v_{1}(m-4,4-m)}{m-4} \\
= & \left.v_{1}(m-4), 4-m\right)+v_{0}(m-4,4-m)+\frac{v_{1}(m-4,4-m)}{m-4} \\
= & m-4+2+1=m-1=u_{2}(m, m)-u_{1}(m, m)=\frac{u_{3}(m, m)}{m} .
\end{aligned}
$$


Also, for $n=2,3, \ldots$ we have

$$
\begin{aligned}
& \frac{v_{2 n+1}(m-4,4-m)}{(m-4)^{n+1}} \\
= & \frac{v_{2 n-1}(m-4,4-m)+v_{2 n}(m-4,4-m)}{(m-4)^{n}} \\
= & \frac{(1+(m-4)) v_{2 n-1}(m-4,4-m)+(m-4) v_{2 n-2}(m-4,4-m)}{(m-4)^{n}} \\
= & \frac{(m-2) v_{2 n-1}(m-4,4-m)-(m-4) v_{2 n-3}(m-4,4-m)}{(m-4)^{n}} \\
= & (m-2) \frac{v_{2 n-1}(m-4,4-m)}{(m-4)^{n}}-\frac{v_{2 n-3}(m-4,4-m)}{(m-4)^{n-1}}
\end{aligned}
$$

and

$$
\begin{aligned}
\frac{u_{2 n+1}(m, m)}{m^{n}} & =\frac{u_{2 n}(m, m)-u_{2 n-1}}{m^{n-1}} \\
& =\frac{(m-1) u_{2 n-1}(m, m)-m u_{2 n-2}(m, m)}{m^{n-1}} \\
& =\frac{(m-1) u_{2 n-1}(m, m)-\left(u_{2 n-1}(m, m)+m u_{2 n-3}(m, m)\right)}{m^{n-1}} \\
& =(m-2) \frac{u_{2 n-1}(m, m)}{m^{n-1}}-\frac{u_{2 n-3}(m, m)}{m^{n-2}} .
\end{aligned}
$$

Thus, by induction (2.5) holds for all $n \in \mathbb{N}$.

(ii) By part (i),

$u_{p}(m, m)=\frac{m^{(p-1) / 2}}{(m-4)^{(p+1) / 2}}\left(v_{p}(m-4,4-m)-(m-4)^{p}\right)+(m(m-4))^{(p-1) / 2}$.

Since $v_{p}(m-4,4-m) \equiv(m-4)^{p}(\bmod p)$ and

$$
\begin{aligned}
& \Delta^{(p-1) / 2}-\left(\frac{\Delta}{p}\right) \\
= & (m-4)^{(p-1) / 2}\left(m^{(p-1) / 2}-\left(\frac{m}{p}\right)\right)+\left(\frac{m}{p}\right)\left((m-4)^{(p-1) / 2}-\left(\frac{m-4}{p}\right)\right) \\
\equiv & \left(\frac{\Delta}{p}\right)\left(\frac{m}{p}\right)\left(m^{(p-1) / 2}-\left(\frac{m}{p}\right)\right) \\
& +\left(\frac{\Delta}{p}\right)\left(\frac{m-4}{p}\right)\left((m-4)^{(p-1) / 2}-\left(\frac{m-4}{p}\right)\right) \\
\equiv & \frac{1}{2}\left(\frac{\Delta}{p}\right)\left(m^{p-1}-1+(m-4)^{p-1}-1\right)\left(\bmod p^{2}\right),
\end{aligned}
$$


we have

$$
\begin{aligned}
u_{p}(m, m)-\left(\frac{\Delta}{p}\right) \equiv & \frac{\left(\frac{m}{p}\right)}{(m-4)\left(\frac{m-4}{p}\right)}\left(v_{p}(m-4,4-m)-(m-4)^{p}\right) \\
& +\frac{1}{2}\left(\frac{\Delta}{p}\right)\left(m^{p-1}-1+(m-4)^{p-1}-1\right) \\
\equiv & \frac{1}{m-4}\left(\frac{\Delta}{p}\right)\left(v_{p}(m-4,4-m)-(m-4)^{p}\right) \\
& +\frac{p}{2}\left(\frac{\Delta}{p}\right)\left(q_{p}(m)+q_{p}(m-4)\right)\left(\bmod p^{2}\right) .
\end{aligned}
$$

On the other hand, by [S10a, Lemma 2.4] we have

$$
2 u_{p}(m, m)-\left(\frac{\Delta}{p}\right) m^{p-1} \equiv u_{p}(m-2,1)+u_{p-\left(\frac{\Delta}{p}\right)}(m-2,1)\left(\bmod p^{2}\right) .
$$

Thus

$$
\begin{aligned}
& \frac{2}{m-4}\left(\frac{\Delta}{p}\right)\left(v_{p}(m-4,4-m)-(m-4)^{p}\right) \\
\equiv & u_{p}(m-2,1)-\left(\frac{\Delta}{p}\right)+u_{p-\left(\frac{\Delta}{p}\right.}(m-2,1)-\left(\frac{\Delta}{p}\right) p q_{p}(m-4)\left(\bmod p^{2}\right) .
\end{aligned}
$$

Comparing this with (2.4) we have reduced (2.4) to the following congruence

$$
u_{p}(m-2,1)-\left(\frac{\Delta}{p}\right) \equiv\left(\frac{m}{2}-1\right) u_{p-\left(\frac{\Delta}{p}\right)}(m-2,1)\left(\bmod p^{2}\right) .
$$

Let $\alpha$ and $\beta$ be the two roots of the equation $x^{2}-(m-2) x+1=0$. Then

$v_{n}(m-2,1)^{2}-\Delta u_{n}^{2}(m-2,1)=\left(\alpha^{n}+\beta^{n}\right)^{2}-\left(\alpha^{n}-\beta^{n}\right)^{2}=4(\alpha \beta)^{n}=4$ for all $n \in \mathbb{N}$. As $u_{p-\left(\frac{\Delta}{p}\right)}(m-2,1) \equiv 0(\bmod p)$ we have

$$
v_{p-\left(\frac{\Delta}{p}\right)}(m-2,1)^{2}-4 \equiv 0(\bmod p) .
$$

By $\left[\right.$ S10a, Lemma 2.3], $v_{p-\left(\frac{\Delta}{p}\right)}(m-2,1) \equiv 2(\bmod p)$. So

$$
v_{p-\left(\frac{\Delta}{p}\right)}(m-2,1) \equiv 2\left(\bmod p^{2}\right) \text {. }
$$

By induction, $(m-2) u_{n}(m-2,1) \pm v_{n}(m-2,1)=2 u_{n \pm 1}(m-2,1)$ for all $n \in \mathbb{Z}^{+}$. Therefore

$$
\begin{aligned}
2 u_{p}(m-2,1) & =(m-2) u_{p-\left(\frac{\Delta}{p}\right)}(m-2,1)+\left(\frac{\Delta}{p}\right) v_{p-\left(\frac{\Delta}{p}\right)}(m-2,1) \\
& \equiv(m-2) u_{p-\left(\frac{\Delta}{p}\right)}(m-2,1)+2\left(\frac{\Delta}{p}\right)(\bmod p)
\end{aligned}
$$

and hence (2.6) follows.

The proof of Lemma 2.4 is now complete.

Combining Lemmas 2.3 and 2.4 we get the following result. 
Lemma 2.5. Let $p$ be an odd prime and let $m \in \mathbb{Z}$ with $\Delta=m(m-4) \not \equiv$ $0(\bmod p)$. Then

$$
\sum_{k=1}^{p-1}(-1)^{k} \frac{\left(\begin{array}{c}
2 k \\
k
\end{array}\right)}{k(m-4)^{k}} \equiv q_{p}(m-4)-\frac{m}{2}\left(\frac{\Delta}{p}\right) \frac{u_{p-\left(\frac{\Delta}{p}\right)}(m-2,1)}{p}(\bmod p) \text {. }
$$

\section{Proofs of Theorems 1.1-1.2 And (1.3)}

Proof of Theorem 1.2. For $k=0, \ldots, p^{a}-1$, clearly

$$
\begin{aligned}
\left(\begin{array}{c}
h p^{a}-1 \\
k
\end{array}\right)(-1)^{k} & =(-1)^{k} \prod_{0<j \leqslant k} \frac{h p^{a}-j}{j}=\prod_{0<j \leqslant k}\left(1-h \frac{p^{a}}{j}\right) \\
& \equiv 1-h \sum_{0<j \leqslant k} \frac{p^{a}}{j}=1-h p^{a} H_{k}(\bmod p) .
\end{aligned}
$$

Thus

$$
\sum_{k=0}^{p^{a}-1}\left(\begin{array}{c}
h p^{a}-1 \\
k
\end{array}\right) \frac{\left(\begin{array}{c}
2 k \\
k
\end{array}\right)}{(-m)^{k}} \equiv \sum_{k=0}^{p^{a}-1} \frac{\left(\begin{array}{c}
2 k \\
k
\end{array}\right)}{m^{k}}-h p^{a} \sum_{k=0}^{p^{a}-1} \frac{H_{k}}{m^{k}}\left(\begin{array}{c}
2 k \\
k
\end{array}\right)(\bmod p) .
$$

If $p \nmid m-4$, then by applying Lemmas $2.1,2.2$ and 2.5 we get

$$
\begin{aligned}
& \sum_{k=0}^{p^{a}-1}\left(\begin{array}{c}
h p^{a}-1 \\
k
\end{array}\right) \frac{\left(\begin{array}{c}
2 k \\
k
\end{array}\right)}{(-m)^{k}} \\
\equiv & \sum_{k=0}^{p^{a}-1} \frac{\left(\begin{array}{c}
2 k \\
k
\end{array}\right)}{m^{k}}+p h\left(\frac{\Delta}{p^{a}}\right) \sum_{k=1}^{p-1}(-1)^{k} \frac{\left(\begin{array}{c}
2 k \\
k
\end{array}\right)}{k(m-4)^{k}} \\
\equiv & \left(\frac{\Delta}{p^{a}}\right)+\left(\frac{\Delta}{p^{a-1}}\right) u_{p-\left(\frac{\Delta}{p}\right)}(m-2,1) \\
& +p h\left(\left(\frac{\Delta}{p^{a}}\right) q_{p}(m-4)-\frac{m}{2}\left(\frac{\Delta}{p^{a-1}}\right) \frac{u_{p-\left(\frac{\Delta}{p}\right)}(m-2,1)}{p}\right)(\bmod p)
\end{aligned}
$$

and hence $(1.7)$ follows. In the case $m \equiv 4(\bmod p)$, we have

$$
p^{a} \sum_{k=1}^{p^{a}-1} \frac{H_{k}}{m^{k}}\left(\begin{array}{c}
2 k \\
k
\end{array}\right) \equiv 2 p \delta_{a, 1}\left(\bmod p^{2}\right)
$$

by Lemma 2.1, and

$$
\begin{aligned}
& u_{p-\left(\frac{\Delta}{p}\right.}(m-2,1)=u_{p}(m-2,1) \\
\equiv & p\left(\frac{m-2}{2}\right)^{p-1}+\delta_{p, 3} m \frac{m-4}{3} \equiv p+\delta_{p, 3}(m-4)\left(\bmod p^{2}\right) .
\end{aligned}
$$


by [S10b, Lemma 2.2]. So (1.7) also holds when $p \mid m-4$.

Since $u_{p-\left(\frac{\Delta}{p}\right)}(m-2,1) \equiv 0(\bmod p)$ by $[$ S10a, Lemma 2.3$],(1.7)$ in the case $h m \equiv 2(\bmod p)$ yields $(1.8)$.

So far we have completed the proof of Theorem 1.2.

Proof of Theorem 1.1. Choose $m \in \mathbb{Z}$ such that $h m \equiv 2\left(\bmod p^{2}\right)$. Clearly $p \nmid m$. Note that

$$
m-4 \equiv \frac{2}{h}-4=\frac{2-4 h}{h}\left(\bmod p^{2}\right)
$$

So we may get (1.1) by applying (1.8). This concludes the proof of Theorem 1.1.

Proof of (1.3). For $k \in \mathbb{N}$ clearly the constant term of

$$
\left(2-x-x^{-1}\right)^{k}=\frac{(-1)^{k}}{x^{k}}(x-1)^{2 k}
$$

is the central binomial coefficient $\left(\begin{array}{c}2 k \\ k\end{array}\right)$. Observe that

$$
\sum_{k=0}^{n-1}\left(\begin{array}{c}
n-1 \\
k
\end{array}\right)(-1)^{k} m^{n-1-k}\left(2-x-x^{-1}\right)^{k}=\left(m-2+x+x^{-1}\right)^{n-1} .
$$

Comparing the constant terms of both sides of the last equality we obtain

$$
\begin{aligned}
& \sum_{k=0}^{n-1}\left(\begin{array}{c}
n-1 \\
k
\end{array}\right)\left(\begin{array}{c}
2 k \\
k
\end{array}\right)(-1)^{k} m^{n-1-k} \\
= & \sum_{k=0}^{\lfloor(n-1) / 2\rfloor}\left(\begin{array}{c}
n-1 \\
k, k, n-1-2 k
\end{array}\right)(m-2)^{n-1-2 k},
\end{aligned}
$$

which is equivalent to (1.3). We are done.

\section{REFERENCES}

[C] L. Calitz, A theorem of Glaisher, Canad. J. Math. 5 (1953), 306-316.

[GZ] V. J. W. Guo and J. Zeng, Some congruences involving central q-binomial coefficients, Adv. in Appl. Math..

[M] F. Morley, Note on the congruence $2^{4 n} \equiv(-1)^{n}(2 n) ! /(n !)^{2}$, where $2 n+1$ is a prime, Ann. Math. 9 (1895), 168-170.

[P] H. Pan, On a generalization of Carlitz's congruence, Int. J. Mod. Math. 4 (2009), 87-93.

[PS] H. Pan and Z. W. Sun, A combinatorial identity with application to Catalan numbers, Discrete Math. 306 (2006), 1921-1940.

[St] R. P. Stanley, Enumerative Combinatorics, Vol. 1, Cambridge Univ. Press, Cambridge, 1999. 
[SSZ] N. Strauss, J. Shallit and D. Zagier, Some strange 3-adic identities, Amer. Math. Monthly 99 (1992), 66-69.

[SS] Z. H. Sun and Z. W. Sun, Fibonacci numbers and Fermat's last theorem, Acta Arith. 60 (1992), 371-388.

[S06] Z. W. Sun, Binomial coefficients and quadratic fields, Proc. Amer. Math. Soc. 134 (2006), 2213-2222.

[S10a] Z. W. Sun, Binomial coefficients, Catalan numbers and Lucas quotients, Sci. China Math., in press. http://arxiv.org/abs/0909.5648.

[S10b] Z. W. Sun, p-adic valuations of some sums of multinomial coefficients, Acta Arith., revised. http://arxiv.org/abs/0910.3892.

[ST1] Z. W. Sun and R. Tauraso, New congruences for central binomial coefficients, Adv. in Appl. Math. 45 (2010), 125-148.

[ST2] Z. W. Sun and R. Tauraso, On some new congruences for binomial coefficients, Int. J. Number Theory, in press. http://arxiv.org/abs/0709.1665. 